\title{
Multiple independent variants in 6q21-22 associated with susceptibility to celiac disease in the Dutch, Finnish and Hungarian populations
}

\begin{abstract}
Elisabet Einarsdottir ${ }^{\star, 1,12}$, Marianna R Bevova ${ }^{2,3,12}$, Alexandra Zhernakova ${ }^{2,4}$, Alienke Monsuur ${ }^{2}$, Lotta LE Koskinen ${ }^{1}$, Ruben van't Slot ${ }^{2}$, Chris Mulder ${ }^{5}$, M Luisa Mearin ${ }^{6}$, Ilma R Korponay-Szabo ${ }^{7}$, Katri Kaukinen $^{8}$, Kalle Kurppa ${ }^{9}$, Juha Kere ${ }^{10}$, Markku Mäki ${ }^{9}$, Cisca Wijmenga ${ }^{11}$ and Päivi Saavalainen ${ }^{1}$

Celiac disease is an inflammatory enteropathy caused by intolerance to gluten. Previous linkage studies in the Dutch, Finnish and Hungarian populations have revealed a locus on chromosome 6q21-22 conferring susceptibility to celiac disease. This locus has previously been implicated in susceptibility to other autoimmune diseases such as Crohn's disease and type 1 diabetes. We performed fine mapping on 446 independent individuals with celiac disease and 641 controls of Dutch origin, testing 872 tagging SNPs in a $22 \mathrm{Mb}$ region of chromosome 6. The 12 most promising SNPs were followed up in 2071 individuals from 284 Finnish and 357 Hungarian celiac disease families to identify risk variants in this region. Multiple markers in the region were significantly associated with celiac disease in the Dutch material. Two SNPs, rs9391227 and rs4946111, were significantly associated with celiac disease in the Finnish population. The association to rs9391227 represents the strongest association signal found in the Finnish $(P=0.003,0 R 0.66)$ as well as the combined Dutch, Finnish and Hungarian populations $\left(P=3.6 \times 10^{-5}, \mathrm{OR} 0.76\right)$. The rs9391227 is situated downstream of the HECT domain and ankyrin repeat containing, E3 ubiquitin protein ligase 1 (HACE1) gene and is contained within a region of strong linkage disequilibrium enclosing HACE1. Two additional, independent, susceptibility variants in the 6q21-22 region were also found in a meta-analysis of the three populations. The 6q21-22 region was confirmed as a celiac disease susceptibility locus and harbors multiple independent associations, some of which may implicate ubiquitin-pathways in celiac disease susceptibility.
\end{abstract}

European Journal of Human Genetics (2011) 19, 682-686; doi:10.1038/ejhg.2011.2; published online 16 February 2011

Keywords: Celiac; linkage; association; genome-wide; 6q21-22

\section{INTRODUCTION}

Celiac disease is a chronic autoimmune disorder of the small intestine with autoimmune features. It is triggered in genetically susceptible individuals by dietary exposure to gluten, a protein present in wheat, barley and rye. Ingestion of gluten by celiac disease patients leads to flattening of the duodenal mucosa, a gradual process classified according to Marsh. ${ }^{1}$ With a prevalence above $1 \%,{ }^{2}$ celiac disease is the most common food intolerance in general Western populations. Long regarded as a gastrointestinal disorder of childhood, the disease is now considered to be a chronic systemic autoimmune disease and is more and more often diagnosed in adults. ${ }^{3}$ Celiac disease is a multifactorial disorder and both environmental factors, such as gluten, and a multigenic predisposition are needed to explain the etiology of the disease. Concordance rates for celiac disease have been shown to be higher in monozygous twins (86\%) than in dizygous twins (20\%), indicating a strong genetic component in the development of celiac disease. ${ }^{4}$ Celiac disease aggregates in families with an $\sim 10 \%$ recur- rence risk for siblings of celiac disease patients. There is, however, no clear Mendelian inheritance of the disease in families, suggesting that the genetic predisposition is derived from the presence and interaction of more than one susceptibility gene.

Over $90 \%$ of celiac disease patients express the HLA-DQ2 heterodimer, encoded by $D Q A 1^{*} 0501$ and $D Q B 1^{*} 02$. A causal role for HLA-DQ2 has been demonstrated as this molecule can present the gluten-derived peptides to T cells. ${ }^{5}$ A further $5 \%$ of celiac disease patients carry HLA-DQ8. Although HLA-DQ2 or DQ8 seem to be necessary for celiac disease development, they are not sufficient, as HLA-DQ2 or DQ8 alone can only explain some $40 \%$ of the genetic variation underlying celiac disease. This implies that other, non-HLA genes, are also important determinants of disease etiology.

A recent whole-genome association scan identified a number of genetic variants involved in celiac disease susceptibility. ${ }^{6}$ However, all the known risk variants explain not more than $50 \%$ of the genetic variance of celiac disease, indicating that novel genes and novel risk

\footnotetext{
${ }^{1}$ Department of Medical Genetics and Research Program for Molecular Medicine, Biomedicum Helsinki, University of Helsinki, Helsinki, Finland; ${ }^{2}$ Department of Medical Genetics, University Medical Centre, Utrecht, The Netherlands; 3Department of Clinical Genetics, VU University Medical Center, Amsterdam, The Netherlands; ${ }^{4}$ Department of Rheumatology, Leiden University Medical Center, Leiden, The Netherlands; ${ }^{5}$ Department of Gastroenterology, VU University Medical Center, Amsterdam, The Netherlands; ${ }^{6}$ Department of Paediatric Gastroenterology, Leiden University Medical Centre, Leiden, The Netherlands; ${ }^{7}$ Heim Pal Children's Hospital, Budapest and University of Debrecen, Debrecen, Hungary; ${ }^{8}$ Department of Gastroenterology and Alimentary Tract Surgery, Tampere University Hospital and Medical School, University of Tampere, Tampere, Finland; ${ }^{9}$ Paediatric Research Centre, University of Tampere Medical School and Tampere University Hospital, Tampere, Finland; ${ }^{10}$ Department of Biosciences and Nutrition, Karolinska Institutet, Stockholm, and Clinical Research Center, Karolinska University Hospital, Huddinge, Sweden; ${ }^{11}$ Department of Genetics, University Medical Center Groningen, and University of Groningen, Groningen, the Netherlands

*Correspondence: Dr E Einarsdottir, Department of Medical Genetics and Research Program for Molecular Medicine, Biomedicum Helsinki, P.O. Box 63, University of Helsinki, Finland. Tel +35 891912 5107; Fax +35 891912 5624; E-mail elisabet.einarsdottir@helsinki.fi

12These authors contributed equally to this work.

Received 19 May 2010; revised 8 December 2010; accepted 5 January 2011; published online 16 February 2011
} 
variants remain to be identified. This was supported by the results of the second whole-genome association study in celiac disease. ${ }^{7}$ This study confirmed a number of the previously identified risk variants, as well as identifying numerous novel variants.

A study in the Dutch population obtained evidence for linkage of celiac disease to the 6q21-22 region, $\sim 70 \mathrm{cM}$ downstream of the HLA region. ${ }^{8}$ The region of interest spans $\sim 102$ to $122 \mathrm{Mb}$ and is clearly distinct from the HLA region as well as from the previously identified TNFAIP 3 and TAGAP celiac susceptibility genes. ${ }^{9,10}$ An independent study by Einarsdottir et al (unpublished) of susceptibility genes for celiac disease in Finnish and Hungarian families also showed linkage to $6 \mathrm{q} 16-22$, to a region overlapping with the linkage found in the Dutch population. Taken together, these results indicate the possibility of a genetic factor within the chromosome 6q21-22 region that is involved in susceptibility to celiac disease in multiple populations. We therefore performed a fine-mapping scan in Dutch unrelated individuals with celiac disease in order to identify the possible candidate gene. This study was further followed up in family data sets from the Finnish and Hungarian populations.

\section{PATIENTS AND METHODS}

\section{Patients and controls}

Dutch cohort. DNA from whole blood was available from a cohort of Dutch individuals with celiac disease. All the affected individuals were diagnosed in accordance with the revised ESPGHAN criteria. ${ }^{11}$ The control cohort is comprised of random blood bank donors and has been described previously. ${ }^{6}$ All 446 Dutch cases and 641 controls were from the Netherlands and of European descent, and at least three of their four grandparents were also born in the Netherlands. The sample set has been described previously. ${ }^{12}$ This study was approved by the Medical Ethical Committee of the University Medical Centre Utrecht.

Finnish and Hungarian cohorts. The Finnish families and cases were collected at the University of Tampere and have been described previously. ${ }^{13}$ The collection of the Hungarian samples has also been described previously. ${ }^{14}$ A total of 1001 individuals from 284 Finnish families (19 unknown phenotype, 359 healthy, 623 affected) and 1070 individuals from 357 Hungarian families (seven unknown phenotype, 471 healthy, 592 affected) were included for genotyping. All sample collections were approved by the ethical committees of the Tampere and Helsinki University Hospitals, Finnish National Public Health Institute, Heim Pal Children's Hospital, Budapest, and the University of Debrecen. All participants were informed about the study according to the study protocol and gave their written informed consent.

\section{Marker selection and genotyping}

For a comprehensive follow-up screen in an independent Dutch data set, we took the lod-1 region (95\% confidence interval; ranging from rs9390679 (101 $261700 \mathrm{bp)}$ ) to rs11154152 (123694693 bp) (National Center for Biotechnology Information NCBI34/hg16)) of the previously identified linkage peak on chromosome $6 .{ }^{15}$ SNPs for fine mapping were selected by downloading all the SNPs typed in the CEPH (Centre d'Etude du Polymorphisme Humain) population (Utah residents with ancestry from Northern and Western Europe) that were located in the genomic sequence of the region of interest from the HapMap database (November 2004, Phase I, http://www.hapmap.org/). The program Tagger (available at http://www.broad.mit.edu/mpg/tagger/) was subsequently used to select tag SNPs to cover the region. We used $r^{2} \geq 0.7$ to perform pairwise tagging. Additionally we set up a minor allele frequency (MAF) cut off $\geq 5 \%$ and took Illumina quality design scores into account for preferential picking of SNPs that are more likely to work on the Illumina platform. The obtained tag SNPs were genotyped using the GoldenGate assay on an Illumina BeadStation 500 GX (Illumina Inc., San Diego, CA, USA). After genotyping all tag SNPs from the initial scan in the Dutch cohort were examined for their resulting quality; all those that were not polymorphic, had a low signal or had clusters that were too wide were excluded (data not shown). In addition, tag SNP that deviated from Hardy-Weinberg equilibrium
(HWE) in the controls $(P<0.001)$ were excluded from further analysis, yielding a final set of 872 tag SNPs obtained for genotype analysis.

For the replication in Finnish and Hungarian cohorts, we chose the 18 markers showing the highest allelic association in the Dutch cohort $(P<0.01)$. Fourteen of the SNPs showed the highest association in the unadjusted model and four additional SNP were chosen from the top markers in a sex adjusted model $(P<0.01)$. As the differences in the analysis were not very large, we have not proceeded further with the sex adjusted model (data not shown). The markers were genotyped by MALDI-TOF analysis on the Massarray Analyzer platform (Sequenom, San Diego, CA, USA). Genotyping was performed at the MAF core facility in Karolinska Institute, Stockholm, Sweden. Genotyping results and sample information were collected in a BC|GENE LIMS database (Biocomputing Platforms, Espoo, Finland). As five markers in the replication study failed (assay design failed or call rate $<90 \%$, data not shown), genotype information for 12 SNP markers was available for analysis.

Deviations from Mendelian inheritance in the family materials were determined with PEDCHECK 1.1. ${ }^{16}$ Mendelian errors were detected in $0.21 \%$ of genotypes in the Finnish samples, and in $0.17 \%$ of the genotypes in the Hungarian samples. All genotypes of a marker were discarded in a family if inheritance errors were detected.

To ensure that the annotation of the rs9391227 SNP on both the Illumina and Sequenom platforms was performed on the same strand, we performed an additional genotyping of rs9391227 on the Taqman platform using 12 controls from Finland (previously genotyped by Sequenom) and 54 Dutch celiac disease cases and controls (previously genotyped by Illumina). All the samples showed $100 \%$ concordance of the genotypes obtained on different platforms (data not shown).

\section{Association analysis}

For the initial association analysis in the Dutch sample set, we performed a single SNP association test using the Haploview program. ${ }^{17}$

Unphased (version 3.0) ${ }^{18}$ (available at http://homepages.lshtm.ac.uk/frank dudbridge/software/unphased/), was used to look for association in families by calculating allele counts in affected offspring and comparing these with counts from untransmitted alleles from parents and unaffected offspring. All affected individuals in a family were used in the association analysis. As each affected individual within a family is not independent, this constitutes in effect, a test of linkage in the presence of possible association.

Unphased was also used to perform a meta-analysis of the Finnish, Hungarian and Dutch sample sets. Population differences were assessed by a global Wald test. All reported $P$-values are two-tailed and uncorrected unless noted. Only single-marker association analysis was performed as the genetic markers in the follow-up study were too distant from each other. Permutation testing (1000 permutations) was used to estimate empirical $P$-values, allowing for multiple testing corrections over all tests performed in a run. PLINK (version 1.06), available at http://pngu.mgh.harvard.edu/ purcell/plink/, was used to perform quantitative trait analysis in order to explore if the associated markers were possible eQTLs for the genes within a $200 \mathrm{~kb}$ window around them.

\section{Power analysis of follow-up study}

The Genetic Power Calculator program (http://pngu.mgh.harvard.edu/ purcell/gpc/) ${ }^{19}$ was used to estimate the power of the Finnish and Hungarian family-based association analysis to replicate the association in the Dutch case-control samples. Parameters for power estimation in the Finnish and Hungarian follow-up materials were estimated from the initial Dutch results and marker rs1998166 as it showed the highest association in the discovery phase. We estimated the power to replicate the association to rs1998166 (protective allele frequency 0.123 , odds ratio 0.616 , and celiac disease prevalence 0.01 ) in the 284 Finnish families to be $64 \%$, and in the 357 Hungarian families to be $74 \%$. When combining the Finish and Hungarian samples we had an estimated power of $93 \%$.

\section{RESULTS}

\section{Dutch association}

In this study, we performed fine mapping of the $6 \mathrm{q}$ linkage region by genotyping 872 SNP markers in 446 cases and 641 controls. 
The association of each SNP to celiac disease is shown in Figure 1 and Supplementary Table 1 . From 872 SNPs, 18 showed association at $P<0.01$ and were included for replication in $C D$ families from Finland and Hungary.

As population sub-structure is a possible cause for false positive results, this issue has been investigated previously for all the Dutch cases and a majority of the controls as a part of another study (Carolien de Kovel, unpublished data). No significant population structure was detected using the STRUCTURE software (http:// pritch.bsd.uchicago.edu/structure.html). The likelihood of increasing numbers of sub-populations decreased continuously, indicating that it is more likely that the individuals were drawn from a single population than from two or more. Also, the assignment of the individuals to the different sub-populations, when the software was forced to create more than one, was never significantly different between cases and controls (Caroline de Kovel, unpublished data). In view of these results, it is unlikely that the results of the association analysis in the Dutch data set were due to population sub-structure.

\section{Follow-up association in finnish and hungarian families}

Table 1 shows the markers studied in the follow-up association study. The empirical 5\% quantile of the best $P$-values for the Finnish data set was $0.0029,0.0033$ for the Hungarian data set, and 0.0027 for the combined Finnish, Hungarian and Dutch data set (the markers with $P$-values lower than these are denoted by ${ }^{*}$ in Table 1$)$. Three SNPs showed consistent replication in either the Finnish or Hungarian families, to the same allele as observed in the Dutch population. Association analysis revealed that the T-allele of rs9391227 was associated with protection from celiac disease in Finnish families ( $P$ 0.003, OR 0.66), as well as allele $\mathrm{T}$ of $\mathrm{rs} 4946111$ ( $P$ 0.007, OR 0.66). Allele $\mathrm{T}$ of rs 9391227 also showed a trend of association with celiac disease in Hungarian families. No other markers were associated with celiac disease in the Hungarian family material.

Association to SNP rs9391227 located downstream of HACE1 and within a region of strong LD surrounding the HACE1 gene was confirmed in a meta-analysis of the Finnish and Hungarian family data sets and the Dutch case-control data set $\left(P 3.625 \times 10^{-5}\right.$, protective allele T OR 0.76). Even if we correct for multiple testing of the 872 SNPs originally tested for, this signal remains significant $(P \mathrm{c}=0.032)$. This represents the strongest association in the combined materials, and it is stronger than in the Dutch case-control data set alone. Two additional markers showed a strong association in the meta-analysis, these were rs1998166 ( $P$ 0.0002, OR 1.56), located in the RWDD1 gene and rs4946578 ( $P$ 0.002, OR 1.25), located upstream from the GJA1 gene. The association signal of these two

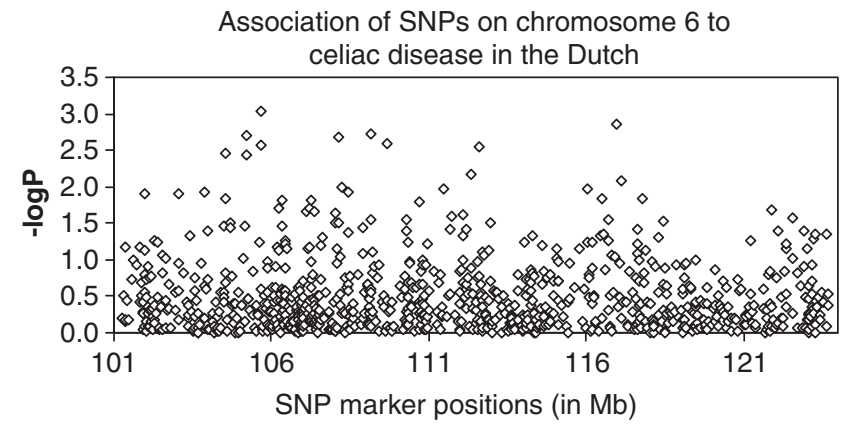

Figure 1 Association of SNPs in the chromosome 6 region to celiac disease in the Dutch cohort. SNP positions are based on NCBI Build 35 and are given in Mb. Diamonds represent individual SNPs.

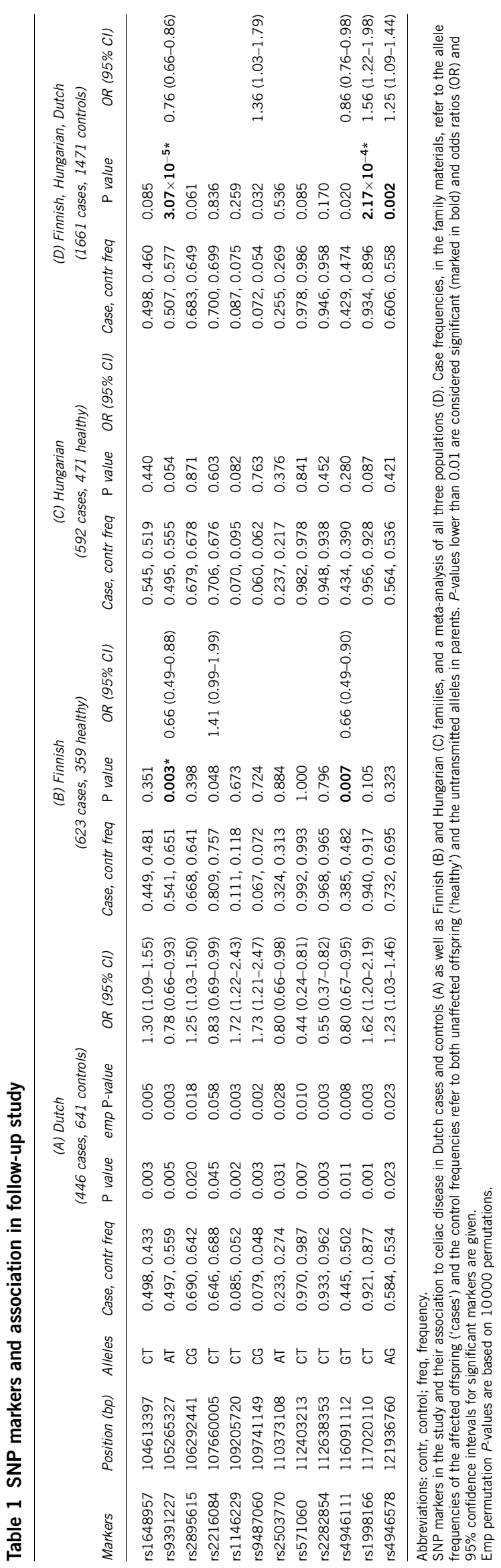


markers to celiac disease appears to be contributed to by all three populations, as the meta $P$ value is lower than that in the Dutch data set only, and the allele frequency differences in patients and controls show the same trend in each of the populations.

Tests of heterogeneity between the data sets yielded significant differences $(P<0.05)$ between populations in the markers rs2216084, rs1146229, rs9487060 and rs4946111. It is expected that there will be significant differences between allele frequencies even in well-sampled data sets from different populations. The markers rs2216084, rs1146229, and rs9487060, while showing some association in the Dutch data set, show very little indication of association in the Finnish and Hungarian data sets. This is not likely to be only due to different allele frequencies as the Dutch and Hungarian data sets show similar frequencies in these markers. Rather, it may be due to different haplotype backgrounds in the different populations. Marker rs4946111 shows some association in the Finnish data set, but less in the Dutch populations. Furthermore, the effect is even reversed in the Hungarian population. This marker may thus constitute a locus on a highly heterogeneous haplotype background between the studied populations. The association in the Finnish samples may be due to an otherwise rare mutation enriched in the Finnish population through genetic drift.

An analysis (data not shown) in 40 Finnish control samples (Dukes et al 2010, unpublished) identified no evidence of eQTLs regarding markers rs9391227 and HACE1, rs1998166 and RWDD1, rs4946578 and GJA1, nor of rs9391227 and PREP expression. PREP is not in LD with rs9391227 (data not shown), further arguing against genetic factors within PREP itself explaining the association with rs9391227. Variation at rs9391227 may still, however, be in LD with and have effects on PREP regulatory regions outside of the gene itself.

\section{DISCUSSION}

In this study we followed up previous findings of linkage to 6q21-22 in celiac disease by performing a fine-mapping study in the Dutch population, and a replication study in the Finnish and Hungarian populations.

\section{HACE1 and rs9391227}

In the combined analysis we identified association of rs9391227 to celiac disease in three populations using both case-control and family data sets. The rs9391227 is an intergenic SNP downstream of and within a region of strong LD surrounding the HACE1 gene (Supplementary Figure 1). HACE1 (HECT domain and ankyrin repeat containing, E3 ubiquitin protein ligase 1) is an ubiquitination-protein ligase. As celiac disease is believed to be due, at least to some extent, to dysregulation of the immune system and antigen presentation, the importance of ubiquitination in celiac disease is plausible. Whether rs9391227 is involved in this mechanism is unknown.

\section{Rs1998166 and rs4946578}

The marker that is second most strongly independently associated with celiac disease in the meta-analysis is rs1998166. This marker is within an intron of the RWD domain containing 1 (RWDD1) gene. Little is known about this gene, but the RWD domain is found in RING finger and WD repeat containing proteins and in the DEXDc-like helicase sub-family, related to the ubiquitin-conjugating enzymes domain.

The third, independent, association found in the meta-analysis was to rs4946578. This marker is $\sim 124 \mathrm{~kb}$ away from, but contained within an LD block that contains the Gap junction protein, alpha 1, $43 \mathrm{kDa}$ (GJA1, CX43) gene. This gene is a member of the connexin gene family and encodes for a component of gap junctions. Connexin-43 is the major protein of gap junctions in the heart, and gap junctions are thought to have a crucial role in the synchronized contraction of the heart and in embryonic development. Mutations in this gene have also been found in deafness ${ }^{20}$ and in palmoplantar keratoderma. ${ }^{21}$

\section{PREP}

The genetic region we studied on $6 \mathrm{q}$ also harbors the gene encoding prolyl endopeptidase (PREP), an enzyme able to cleave proline-rich gluten peptides. An altered PREP activity in the intestinal mucosa could be responsible for the inefficient breakdown of gluten peptides, thus contributing to the onset of celiac disease. PREP has previously been identified as a potential celiac disease candidate; however, a previous study indicated no association of this gene with celiac disease in the Dutch population. ${ }^{22}$ Although this gene is located within the region studied in the current Dutch, Finnish and Hungarian samples, no indication was found that this gene could explain the linkage and/or association to $6 \mathrm{q}$.

\section{Multiple associations and implications}

Linkage to 6q21-22 was found in two independent whole-genome linkage studies, and following up the results, we found association to multiple independent SNPs in the Dutch, Finnish, and Hungarian populations. This replication in several independent data sets makes the association signals in the region credible and likely to be true celiac disease risk factors. We did not find one single gene with a major effect on celiac disease susceptibility, suggesting that there may be several susceptibility genes at this locus, each with small effects. The previously conducted GWAS on celiac disease failed to identify strong associations to celiac disease in the studied region on chromosome 6 . There are eight SNPs among the top 1000 signals from the GWAS of Dubois et al, ${ }^{7}$ which are located in this region, These association signals, however, are in distinct $L D$ blocks $\left(D^{\prime} \leq 0.2\right)$ from the two most highly associated markers in our study (data not shown). Furthermore, the current study is estimated to adequately tag the eight GWAS signals (data not shown). Of the markers in our study, only three (rs1146229, rs2282854, and rs4946578) were also included in the Dubois GWAS study, allowing for a direct comparison of association signals. These three markers were neither significantly associated with celiac disease in the meta-analysis within the Dubois GWAS study nor in the Finnish or Dutch populations (data not shown), these markers were not part of the follow-up study. In addition to this, the arrays used in the GWAS do not contain perfect proxies for the most highly associated markers in our study (data not shown), arguing that the association we see in the current study may have been missed in the GWAS. We thus conclude that our results likely indicate true association that is independent of the GWAS signals, and that it is unlikely that we are missing major associations at this locus due to insufficient marker coverage.

The current study cannot fully explain the underlying cause of the multiple independent association signals in the region. The multiple association signals show the complexity of disease susceptibility in the region, but the results are consistent in the different study populations and the observed risk effect of the associated variants is relatively high. This argues that each of the identified variants (or nearby untyped variants in $\mathrm{LD}$ with the associated variants) is a true, novel, susceptibility factor for celiac disease.

\section{CONFLICT OF INTEREST}

The authors declare no conflict of interest. 


\section{ACKNOWLEDGEMENTS}

All the study subjects are thanked for participating in the study. We thank the Dutch Celiac Disease Foundation for participating in this study, and $\mathrm{H}$ van Someren and F Mulder for clinical database. We thank Wieke HM Verbeek for help in sample collection and Jackie Senior for critical reading of the manuscript. We thank Hanne Ahola and Anne Heimonen for excellent technical assistance and Erzsébet Szathmári, Judit BKovács, Margit Lörincz and Anikó Nagy for their work with the Hungarian families. We thank Carolien de Kovel at UMC Utrecht for providing unpublished data on the population structure of the Dutch cohort. We acknowledge funding from the Coeliac Disease Consortium (an innovative cluster approved by the Netherlands Genomics Initiative and partly funded by the Dutch Government, grant BSIK03009 to CW), the Netherlands Organization for Scientific Research (VICI grant 918.66.620 to CW) and the Dutch Digestive Disease Foundation. This work has been funded by the EU Commission through a Marie Curie Excellence Grant (FP6 contract MEXT-CT-2005-025270 to PS), by the Academy of Finland, the Hungarian Scientific Research Fund (contract OTKA 61868), the University of Helsinki Funds, Biocentrum Helsinki, the Competitive Research Funding of the Tampere University Hospital, and the Foundation of Pediatric Research and the Sigrid Juselius Foundation.

1 Marsh MN: Gluten, major histocompatibility complex, and the small intestine. A molecular and immunobiologic approach to the spectrum of gluten sensitivity ('celiac sprue'). Gastroenterology 1992; 102: 330-354.

2 Lohi S, Mustalahti K, Kaukinen $\mathrm{K}$ et al: Increasing prevalence of coeliac disease over time. Aliment Pharmacol Ther 2007; 26: 1217-1225.

3 Ravikumara M, Tuthill DP, Jenkins HR: The changing clinical presentation of coeliac disease. Arch Dis Child 2006; 91: 969-971.

4 Greco L, Romino R, Coto I et al: The first large population based twin study of coeliac disease. Gut 2002; 50: 624-628.

5 Molberg 0 , Mcadam SN, Korner R et al: Tissue transglutaminase selectively modifies gliadin peptides that are recognized by gut-derived T cells in celiac disease. Nat Med 1998; 4: 713-717.

6 van Heel DA, Franke L, Hunt KA et al: A genome-wide association study for celiac disease identifies risk variants in the region harboring IL2 and IL21. Nat Genet 2007; 39: $827-829$.
7 Dubois PC, Trynka G, Franke L et al: Multiple common variants for celiac disease influencing immune gene expression. Nat Genet 2010; 42: 295-302.

8 Van Belzen MJ, Meijer JW, Sandkuijl LA et al: A major non-HLA locus in celiac disease maps to chromosome 19. Gastroenterology 2003; 125: 1032-1041.

9 Hunt KA, Zhernakova A, Turner G et al: Newly identified genetic risk variants for celiac disease related to the immune response. Nat Genet 2008; 40: 395-402.

10 Trynka G, Zhernakova A, Romanos J et al: Coeliac disease associated risk variants in TNFAIP3 and REL implicate altered NF-kappaB signalling. Gut 2009; 58: 1078-1083.

11 Working group of European Society of Paediatric Gastroenterology and Nutrition. Anonymous revised criteria for diagnosis of coeliac disease. Report of working group of european society of paediatric gastroenterology and nutrition. Arch Dis Child 1990; 65: 909-911.

12 Monsuur AJ, de Bakker PI, Alizadeh BZ et al: Myosin IXB variant increases the risk of celiac disease and points toward a primary intestinal barrier defect. Nat Genet 2005; 37: 1341-1344.

13 Koskinen LL, Einarsdottir E, Dukes E et al: Association study of the IL18RAP locus in three European populations with coeliac disease. Hum Mol Genet 2009; 18: 1148-1155.

14 Koskinen LL, Korponay-Szabo IR, Viiri $\mathrm{K}$ et al: Myosin IXB gene region and gluten intolerance: linkage to coeliac disease and a putative dermatitis herpetiformis association. J Med Genet 2008; 45: 222-227.

15 van Belzen MJ, Vrolijk MM, Meijer JW et al: A genomewide screen in a four-generation Dutch family with celiac disease: evidence for linkage to chromosomes 6 and 9. Am J Gastroenterol 2004; 99: 466-471.

16 O'Connell JR, Weeks DE: PedCheck: a program for identification of genotype incompatibilities in linkage analysis. Am J Hum Genet 1998; 63: 259-266.

17 Barrett JC, Fry B, Maller J, Daly MJ: Haploview: analysis and visualization of LD and haplotype maps. Bioinformatics 2005; 21: 263-265.

18 Dudbridge F: Pedigree disequilibrium tests for multilocus haplotypes. Genet Epidemiol 2003; 25: 115-121.

19 Purcell S, Cherny SS, Sham PC: Genetic power calculator: design of linkage and association genetic mapping studies of complex traits. Bioinformatics 2003; 19: 149-150.

20 Paznekas WA, Karczeski B, Vermeer S et al: GJA1 mutations, variants, and connexin 43 dysfunction as it relates to the oculodentodigital dysplasia phenotype. Hum Mutat 2009; 30: 724-733.

21 van Steensel MA, Spruijt L, van der Burgt I et al: A 2-bp deletion in the GJA1 gene is associated with oculo-dento-digital dysplasia with palmoplantar keratoderma. Am J Med Genet A 2005; 132A: 171-174.

22 Diosdado B, Stepniak DT, Monsuur AJ et al: No genetic association of the human prolyl endopeptidase gene in the Dutch celiac disease population. Am J Physiol Gastrointest Liver Physiol 2005; 289: G495-G500.

Supplementary Information accompanies the paper on European Journal of Human Genetics website (http://www.nature.com/ejhg) 